\title{
Erratum to: Generalizing the Kantorovich Metric to Projection Valued Measures
}

\section{Trubee Davison ${ }^{1}$}

Published online: 18 April 2015

(C) Springer Science+Business Media Dordrecht 2015

Erratum to: Acta Appl. Math. (2014)

DOI 10.1007/s10440-014-9976-y

There is an error in the proof of Theorem 2.15 in the article Generalizing the Kantorovich Metric to Projection Valued Measures [1]. The result is still true. The theorem is restated below:

Theorem 2.15[1] The map $\Phi: P(X) \rightarrow P(X)$ given by

$$
E(\cdot) \mapsto \sum_{i=0}^{N-1} S_{i} E\left(\sigma_{i}^{-1}(\cdot)\right) S_{i}^{*}
$$

is a Lipschitz contraction in the $\rho$ metric.

The following is a corrected version of the part of the proof which contains the error. The author wants to acknowledge Krystal Taylor (University of Minnesota) for identifying the error.

The following argument replaces the argument which begins on the line after Claim 2.16. Let $E, F \in P(X)$. Recall that $r=\max _{0 \leq i \leq N-1}\left\{r_{i}\right\}$, where $r_{i}$ is the Lipschitz constant associated to $\sigma_{i}$, and note that $0<r<1$. Choose $\phi \in \operatorname{Lip}_{1}(X)$, and $h \in \mathcal{H}$ with $\|h\|=1$. Then

$$
\left|\left\langle\left(\int \phi d \Phi(E)-\int \phi d \Phi(F)\right) h, h\right\rangle\right|
$$

The online version of the original article can be found under doi:10.1007/s10440-014-9976-y.

\footnotetext{
T. Davison

trubee.davison@colorado.edu

1 Department of Mathematics, University of Colorado, Boulder, USA
} 


$$
\begin{aligned}
& =\left|\left\langle\left(\int \phi d \Phi(E)\right) h, h\right\rangle-\left\langle\left(\int \phi d \Phi(F)\right) h, h\right\rangle\right|=\left|\int_{X} \phi d \Phi(E)_{h, h}-\int_{X} \phi d \Phi(F)_{h, h}\right| \\
& =\left|\sum_{i=0}^{N-1} \int_{X} \phi d E_{S_{i}^{*} h, S_{i}^{*} h}\left(\sigma_{i}^{-1}(\cdot)\right)-\sum_{i=0}^{N-1} \int_{X} \phi d F_{S_{i}^{*} h, S_{i}^{*} h}\left(\sigma_{i}^{-1}(\cdot)\right)\right| \\
& =\left|\sum_{i=0}^{N-1} \int_{X}\left(\phi \circ \sigma_{i}\right) d E_{S_{i}^{*} h, S_{i}^{*} h}-\sum_{i=0}^{N-1} \int_{X}\left(\phi \circ \sigma_{i}\right) d F_{S_{i}^{*} h, S_{i}^{*} h}\right| \\
& =\left|\sum_{i=0}^{N-1}\left(\int_{X}\left(\phi \circ \sigma_{i}\right) d E_{S_{i}^{*} h, S_{i}^{*} h}-\int_{X}\left(\phi \circ \sigma_{i}\right) d F_{S_{i}^{*} h, S_{i}^{*} h}\right)\right| \\
& =\left|\sum_{i=0}^{N-1} r\left(\int_{X}\left(\frac{\phi \circ \sigma_{i}}{r}\right) d E_{S_{i}^{*} h, S_{i}^{*} h}-\int_{X}\left(\frac{\phi \circ \sigma_{i}}{r}\right) d F_{S_{i}^{*} h, S_{i}^{*} h}\right)\right| \\
& \leq r\left(\sum_{i=0}^{N-1}\left|\int_{X}\left(\frac{\phi \circ \sigma_{i}}{r}\right) d E_{S_{i}^{*} h, S_{i}^{*} h}-\int_{X}\left(\frac{\phi \circ \sigma_{i}}{r}\right) d F_{S_{i}^{*} h, S_{i}^{*} h}\right|\right) \\
& =r\left(\sum_{i=0}^{N-1}\left|\left\langle\left(\int\left(\frac{\phi \circ \sigma_{i}}{r}\right) d E-\int\left(\frac{\phi \circ \sigma_{i}}{r}\right) d F\right) S_{i}^{*} h, S_{i}^{*} h\right\rangle\right|\right) \\
& \leq r\left(\sum_{i=0}^{N-1}\left\|\int\left(\frac{\phi \circ \sigma_{i}}{r}\right) d E-\int\left(\frac{\phi \circ \sigma_{i}}{r}\right) d F\right\|\left\|S_{i}^{*} h\right\|^{2}\right) .
\end{aligned}
$$

Note that the function $\frac{\phi \circ \sigma_{i}}{r} \in \operatorname{Lip}_{1}(X)$ for all $0 \leq i \leq N-1$. Hence

$$
\begin{aligned}
& r\left(\sum_{i=0}^{N-1}\left\|\int\left(\frac{\phi \circ \sigma_{i}}{r}\right) d E-\int\left(\frac{\phi \circ \sigma_{i}}{r}\right) d F\right\|\left\|S_{i}^{*} h\right\|^{2}\right) \\
& \leq r \rho(E, F)\left(\sum_{i=0}^{N-1}\left\langle S_{i}^{*} h, S_{i}^{*} h\right\rangle\right)=r \rho(E, F)\left(\sum_{i=0}^{N-1}\left\langle S_{i} S_{i}^{*} h, h\right\rangle\right) \\
& \quad=r \rho(E, F)\left\langle\left(\sum_{i=0}^{N-1} S_{i} S_{i}^{*}\right) h, h\right\rangle=r \rho(E, F)\langle h, h\rangle=r \rho(E, F) .
\end{aligned}
$$

Therefore

$$
\left\|\int \phi d \Phi(E)-\int \phi d \Phi(F)\right\| \leq r \rho(E, F) .
$$

Since $\phi$ is an arbitrary element of $\operatorname{Lip}_{1}(X)$,

$$
\rho(\Phi(E), \Phi(F)) \leq r \rho(E, F) .
$$

This proves that $\Phi$ is a Lipschitz contraction in the $\rho$ metric on $P(X)$. 


\section{References}

1. Davison, T.: Generalizing the Kantorovich metric to projection-valued measures. Acta Appl. Math. (2014). doi:10.1007/s10440-014-9976-y 